\title{
Frequency Stability of the European Interconnected Power System Under Grid Splitting in Market Zones
}

\author{
Carmelo Mosca $^{1, *}$, Ettore Bompard ${ }^{1}$, Gianfranco Chicco ${ }^{1}$, João Moreira ${ }^{2}$, Vincent Sermanson ${ }^{2}$, Dante Powell ${ }^{2}$
}

\author{
${ }^{1}$ Dipartimento Energia "Galileo Ferraris", Politecnico di Torino, Torino, Italy \\ ${ }^{2}$ Drafting Team Planning Standards, ENTSO-E, Bruxelles, Belgium
}

\begin{abstract}
This paper proposes a graph theory-based approach to define the possible separation of the market zones in large power systems. The market zone partitioning is used to assess the frequency stability based on a set of parameters, including the inertia, the running capacity of the separated areas, and the power exchanged on the interconnection lines. A system split indicator is finally used to rank the worst split lines. The methodology has been tested on real scenarios of the interconnected Continental Europe power system.
\end{abstract}

Index Terms: frequency stability, grid separation, market zones, power system inertia, system split.

\section{INTRODUCTION}

The energy policy framework adopted by the European Union to facilitate the transition from fossil fuels towards cleaner renewable energy sources (RES) is drastically changing the European interconnected power system [1]. Grid reinforcement, investment and new policies are needed to allocate a high level of RES, to reduce market electricity prices, and to increase the security of supply in future scenarios with a low number of conventional generating units, bringing to high capacity exchange between the national power systems. At the same time, RES are typically interconnected to the power system through power electronic devices, decoupling the generator from the grid without contributing to the overall system inertia [2]. Furthermore, the power electronic devices cannot provide inherently support to the grid, in terms of ancillary services for frequency and voltage stability, jeopardizing the system stability [3]. In this context, ensuring power

\footnotetext{
${ }^{*}$ Corresponding author.

E-mail: carmelo.mosca@polito.it
}

http://dx.doi.org/10.38028/esr.2020.04.0005

Received October 16, 2020. Revised November 11, 2020.

Accepted December 03, 2020. Available online February 01, 2021.

This is an open access article under a Creative Commons Attribution-NonCommercial 4.0 International License.

(C) 2020 ESI SB RAS and authors. All rights reserved. system stability requires a coherent set of actions and mitigation solutions at the Transmission System Operator (TSO) level, across neighbors, or at synchronous area level, depending on different stability phenomena. Frequency stability requires a synchronous area perspective, and the frequency containment process is specified by the European Commission Guidelines [4]. These Guidelines are designed according to the reference incident of 3 GW for the Continental Europe (CE) synchronous area. Several solutions have been investigated to support frequency stability in the case of low inertia, ranging from physical components (as energy storage systems or synchronous compensators [5]) or market modifications, as well as the addition of inertia constraints in the unit commitment phase [6]. Other studies have been conducted to determine the critical power imbalances and maximum admissible rate of frequency change showing that for the $\mathrm{CE}$ synchronous system, the reference incident does not imply particular concerns, at least for the overall frequency stability [7]. However, the past system splits outlined the possibility of imbalance higher than the reference incident and, especially, in smaller regions. In the case of multiple line outages, cascading line failures can separate synchronously interconnected transmission grid into two or more asynchronous areas. In these cases, the frequency stability of the system is endangered, given the increasing power exchange between various regions resulting in larger power imbalances, and the decreasing system inertia leading to higher frequency gradients [8].

The bulk transmission system can be represented as a graph, with physical nodes and branches. Starting from this representation, simplifications have been proposed to reduce the complexity of large-scale systems [9]. Highlevel major nodes may be formed, where each node contains a subsystem whose internal connections do not limit power exchanges [10]. From another point of view, major nodes may be formed by using clustering algorithms [11] to represent bidding zones in the prospect of zonal electricity markets [12]. These high-level major nodes are then connected to design simpler graph structures.

Currently, there are a few studies on the definition of a methodology for the identification of system splits. Some 
studies were aimed at determining the splitting boundary to damp system oscillations post-fault. A method for searching those splitting boundaries is presented in [13] to minimize the load-generation imbalance in each island. Spectral partitioning is used in [14] to determine the weak links based on topologic considerations for a static system. An approach to the systematic identification of critical system split topologies is described in [15], with the definition of relevant initial contingencies corroborated by time-domain simulations to determine the actual cascading line failure leading to a system split. The identification of a cutset for a large-scale power system is presented in [9] for the application to controlled islanding aimed at preventing the effects of cascading outages.

In this paper, the identification of system split is based on the consideration of the market exchanges between the European market zones in the context of future scenarios developed in the Ten-Year Network Development Plan (TYNDP) by ENTSO-E. A graph theory approach is used to calculate all the possible system splits into two asynchronous areas and their inertia is used to calculate the frequency performance of the system in terms of the Rate of Change of Frequency (ROCOF). The worst split lines are ranked using a system split indicator. The obtained results can be used by the TSOs to take eventual mitigating actions, in terms of grid reinforcement, regulations, or new protection schemes. This is a novel view on the existing approaches.

The outline of this paper is as follows: Section II gives a brief overview of the frequency regulation schemes adopted in Europe, the process of the system development in the framework of the TYNDP, and an overview of past system split in the world. Section III considers the implemented methodology to identify possible system splits and describes the process and the inertia calculation method. In Section IV, the methodology is applied in future scenarios of the European interconnected power system. The last section contains the conclusions.

\section{EUROPEAN FREQUENCY DYNAMICS AND PLANNING}

\section{A. Frequency dynamics in power systems}

Frequency variations occur in power systems due to mismatches between active power generation and demand. After such mismatch, the energy stored in the rotating masses of the synchronous generating units instantaneously provides balance actions through the inertial response, resulting in a change in rotor speed and the system frequency. Inertia is essential for the first instantaneous balance between generation and demand, even if it is not enough to restore the system frequency, power units capable of varying their power output according to the frequency changes are required, giving the primary frequency regulation. If a power imbalance occurs, each generator in the power system follows a different oscillatory motion around the center of inertia.
Nevertheless, all individual machines can be aggregated into a single unit, whose mechanical behavior is governed by a single swing equation to represent the response of all generators:

$$
\frac{d f}{d t}=\frac{f_{0}}{2 H_{s y s} S_{t o t}}\left(\Delta P_{m}-\Delta P_{L}+D \cdot \Delta f\right),
$$

where $\Delta P_{m}$ is the mechanical power produced by the regulating resources in the grid; $f_{0}$ is the nominal frequency, $H_{s y s}$ is the aggregated inertia of the system; $S_{t o t}$ is the total rated power of the generators, and $D$ represents the load damping coefficient due to the load frequency dependency.

In a synchronous zone, the power-demand variations are addressed by the Load Frequency Control (LFC) scheme. This scheme comprises, in a temporal sequence, the Frequency Containment Process (FCP, primary) automatically activated to stabilize the frequency deviation using the Frequency Containment Reserve (FCR, secondary); the Frequency Restoration Process (FRC) automatically and manually activated in the area where the imbalance occurs to return the frequency to its nominal value using the Frequency Restoration Reserve (FRR); and the Replacement Reserve Process (RRP, tertiary) manually activated to replace the activated FRR using the Replacement Reserve (RR). The LFC has its characteristics and qualities for each synchronous zone. ENTSO-E grid is composed of 8 synchronous zones: Continental Europe, Baltic, Nordic, British, Irish, Sardinia-Corsica, Cyprus, and Crete. Each synchronous area is presented in Fig. 1 with the belonging countries. In this paper, the focus is on the $\mathrm{CE}$ synchronous zone.

\section{B. Ten Year Network Development Plan (TYNDP)}

The TYNDP is published by ENTSO-E every two years to present how the grid is going to develop in the next 10 to 20 years and how the grid development can effectively contribute to achieving different and sometimes competing goals set by the European Energy Transition [16]. The main role of the TYNDP is to identify where investment in the electricity system would help deliver the Energy Union and benefit all Europeans. This has been done in two stages: (I) starting with a theoretical overview of the optimal set-up allowing for the decarbonization of the EU power system at the lowest cost (system needs analysis), and (II) a call for transmission and storage projects (under different stages of development) across Europe and complemented by an analysis of their performance under the different scenarios. Scenarios describe possible European energy futures up to 2050 but are not forecasts: they set out a range of possible futures used to test foreseen electricity and gas infrastructure needs and projects. The TYNDP starts with the development of scenarios or visions of the future European power system (e.g., in 2030 and 2040). Scenarios are a prerequisite for any study analyzing the future of the European energy system, and they are developed by ENTSO-E and its gas counterpart ENTSO-G. Each scenario's impacts on energy markets and 


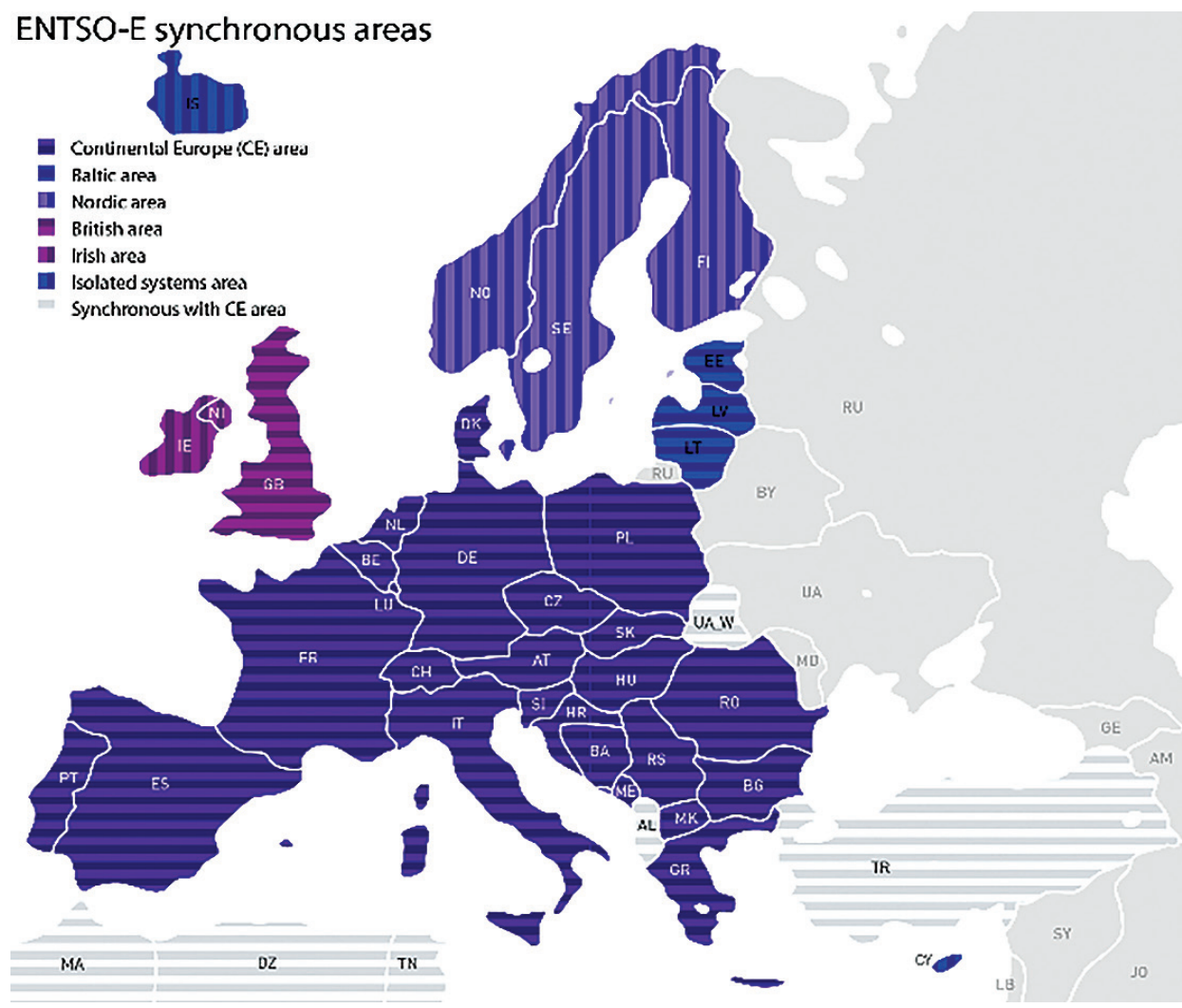

Fig. 1. ENTSO-E synchronous areas.

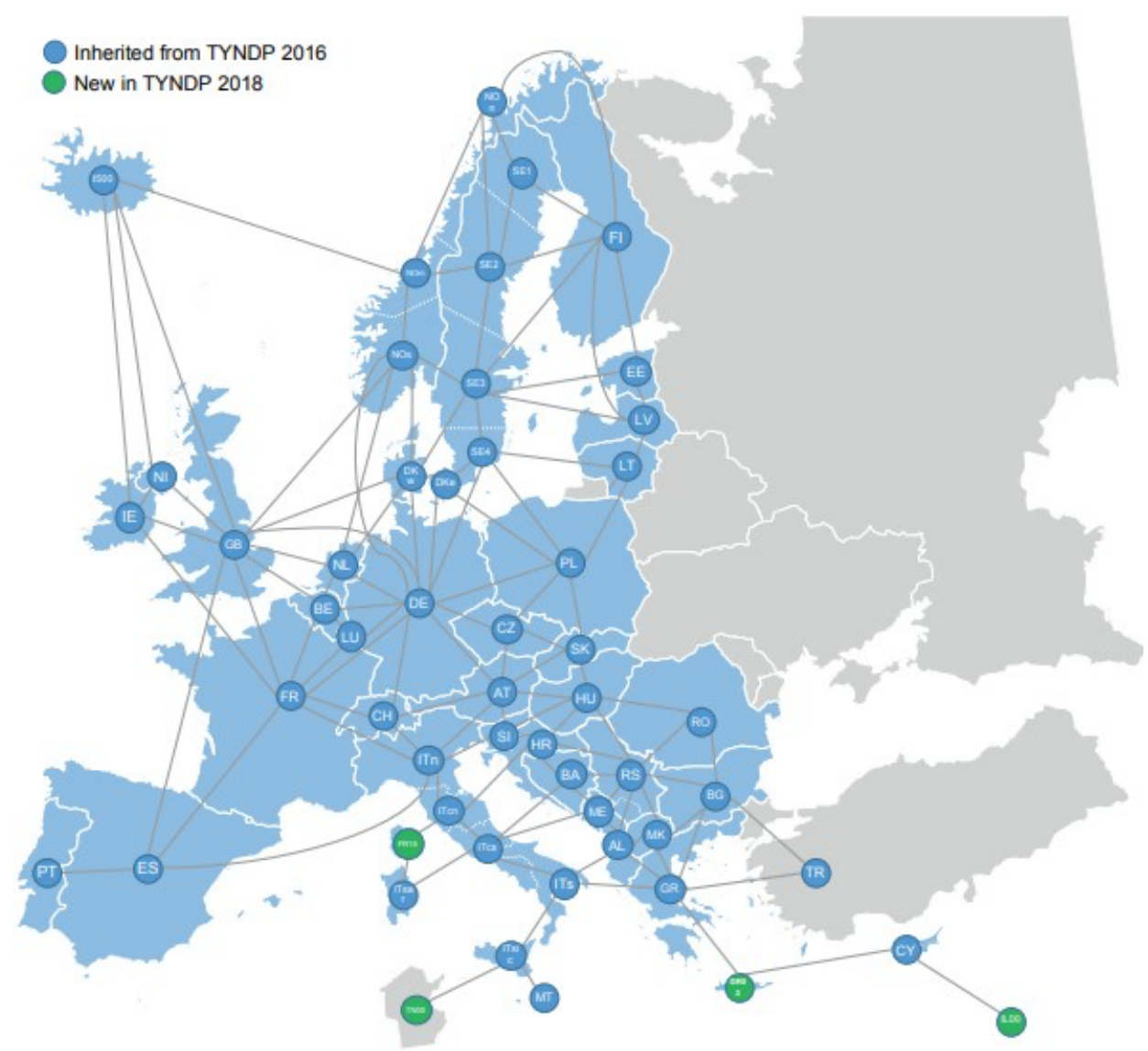

Fig. 2. Market zones from the TYNDP18. 
networks are analyzed with the help of tailored modeling tools for the identification of system needs (IoSN) and to understand which parts of the network infrastructure are working well, and where it needs to be stronger [17]. For each scenario, the system needs are identified using market and network simulations. The market simulation outputs represent the input for the IoSN network simulation, to analyze possible bottlenecks and identify projects which would benefit the system in a feasible and cost-efficient manner. Market simulations are performed using different market zones and the ones used for the TYNDP 2018 are shown in Fig. 2. The European bulk power system is divided into different interconnected market zones, which are characterized by interchanged transfer capacities.

The ENTSO-E has agreed common definitions for these exchanges: Net Transfer Capacity (NTC), Available Transfer Capacity (ATC), Transmission Reliability Margin (TRM), and Already Allocated Capacity (AAC) [18]. NTC and ATC are an important basis for the market to anticipate and plan cross-border transactions and for the TSOs to manage the electricity exchanges. NTC calculations require that TSOs perform extensive studies of load flows in the interconnected European transmission system. The NTC is interpreted as the expected maximum volume of power that can be exchanged through the interface between two systems, which does not lead to violation of network constraints in either system, respecting some technical uncertainties on future network conditions. Future market simulation tools use the future NTC as a constraint to calculate the AAC for each hour of the year. In this work, the AAC is used as the possible imbalance that could follow a system split. TSOs need to systematically assess the long-term changes in various operational parameters such as inertia and short-circuit current levels, operational requirements such as flexibility, and availability of ancillary services such as reactive power support, frequency response, and contribution to short-circuit current [19].

TYNDP recently started exploring real-time system operation needs (voltage and frequency control) in response to new challenges expected to grow in the future because of the changing energy generation mix and increasingly responsive energy demand [20], [21]. Focusing on the frequency stability, small synchronous areas would see rapid and large frequency excursions following a normal generation loss, large synchronous areas would not see the same size of frequency excursions unless a significant disturbance occurs such as a system split.

\section{Overview of major system split events}

The separation of the European synchronously interconnected power system into two or more asynchronous areas represents one of the major concerns for the system stability. This section discusses the state-of-the-art of system split concepts and presents a brief overview of the past system splits, which occurred throughout the world.
In a system split event, the synchronous area splits into separate islands. The exports and imports between these islands before the system split event turn into power imbalances for the separate islands after the split. The larger the export or import of the island before the split, the greater the imbalance after the split and, therefore, the greater the need for large and quick adjustment for generation and demand. Not only the resulting imbalances are difficult to predict but also the resulting equivalent system inertia will differ from island to island. Based on scheduled grid expansion measures for future grid development scenarios, corresponding market simulations, and dynamic system studies, the ENTSO-E proposes a maximum admissible power imbalance of $40 \%$ per region within CE and a maximum admissible frequency gradient of $2 \mathrm{~Hz} / \mathrm{s}$. This generalized approach aims at covering any system split scenario without conducting a detailed analysis of the potential split topologies [7]. However, under some conditions, it is reasonable to consider the existence of large initial ROCOFs exceeding $2 \mathrm{~Hz} / \mathrm{s}$. A system split is more prone to occur across congested transit corridors and thus interrupting these transits. As transits are increasing in magnitude, distance, and volatility, the power imbalance following a system split event is likely to increase. This would consequently lead to larger, longer, and quicker frequency excursions in subsequently formed islands. The increased imbalance must be compensated by fast frequency response including fast control reserves or frequency-related defense measures, for example, LimitedFrequency-Sensitive-Mode Over-frequency (LFSMO) or Low-Frequency Demand Disconnection (LFDD). According to the system defense operation guidelines, a system split will result in an emergency state because of out-of-range contingency. TSOs will not act preventively to mitigate the impact of out of range contingency but will react by activating their defense plan. Defense plans are designed to help during those severe disturbances but cannot stabilize all system split scenarios with extreme imbalances. Potentially needed restoration plans will employ adequate resources to stabilize the islands and later to re-synchronize the system.

Various blackouts occurred in different parts of the world [22], among which the major system disturbances in CE that occurred on September 28th, 2003, with the Italian system separation [23], and on November 4th, 2006 [8]. After the major disturbances in 2003 and 2006, the third serious event in the CE system was a blackout in Turkey on March 31st, 2015 [24]. All these events had similar characteristics such as high corridor loading, underfrequency load shedding, and non-conforming power plant behavior with respect to abnormal frequency deviations. Two major blackouts occurred in Brazil on November 10th, 2009, and on February 4th, 2011, involving, respectively, the disconnection of a $\mathrm{HV}$ transmission line and a $\mathrm{HV}$ substation. In both cases, cascading failures following the main events led first to regions islanding and later to 
the system collapse [25]. On July 30th, 2012, the Indian power system suffered a severe disturbance, which led to a blackout initiated by overloading of an inter-regional tie line and followed by cascading failures and separation of the interconnected regions [26]. On July 12, 2004, the south part of the Hellenic Interconnected Transmission System (including Athens) was split from the rest of the system and collapsed, which was initiated by the opening of a north-south HV transmission line [27].

\section{System Split Methodology}

\section{A. Inertia calculation from market studies}

Before introducing a system split identification methodology, it is necessary to describe the procedure for the inertia calculations. Generally, the power system planning process requests data from all the TSOs for different studies. Different future scenarios are considered, which include installed capacities, demand, and crossborder capacities. For each scenario, a market simulation is performed, the market modeling outputs are given with an hourly granularity and show the hourly dispatch for each unit's type and for the countries in the interconnected areas. The requested data for frequency studies are the typical values of inertia $H_{g, i}$ provided by TSOs per fuel type $g$ and the nominal capacity $P_{g n, i}$ of the generator $i$ for all the $N$ synchronous plants in the country. This information is organized into subcategories based on technology type, from which the average inertia constant $H_{g}$ and the reference average capacity $P_{g n}$ for each type of synchronous units are established per country:

$$
\begin{gathered}
H_{g}=\frac{\sum_{i=1}^{N} H_{g, i} P_{g n, i}}{\sum_{i=1}^{n} P_{g n, i}}, \\
P_{g n}=\frac{\sum_{i=1}^{N} P_{g n, i}}{N} .
\end{gathered}
$$

The market modeling simulation gives the total generated power in [MW] for each hour $h$ and fuel type per country $P_{g}(h)$. The number of units $n$ running for each technology can be estimated by using the reference average capacity of a unit:

$$
n_{g}(h)=\frac{P_{g}(h)}{P_{g n} \cdot l_{g}},
$$

where $l_{g}$ is the loading factor per country and for the generator technology type $g$. The loading factor is the ratio between the generated energy in a year, divided by the energy the plant would have produced when generating at maximum power. Generally, high loading factors characterize nuclear and conventional power plants, while lower values characterize RES. The number of units is rounded up to have an integer value and to be precautionary. In this way, it is possible to calculate the inertia for one specific hour $h$ in a specific zone $z$ using the number of dispatched units multiplied by the average capacity and the inertia constant of the unit type:

$$
H_{z}(h)=\frac{E_{k, z}}{P_{t o t, z}}=\frac{\sum_{g=1}^{G} H_{g, z} P_{g n, z} n_{g, z}}{\sum_{g=1}^{G} P_{g n, z}},
$$

where $E_{k, z}$ is the kinetic energy and $P_{t o t, z}$ the total running capacity of the zone $z$ at hour $h$.

The estimated inertia is calculated based on the online generator's capacity, neglecting the contribution from the demand, and RES is considered not contributing to the inertia.

The initial ROCOF and the magnitude of the frequency deviation depend on the imbalance between generation and demand compared to the total kinetic energy and the frequency dependency of the load, based on the swing equation (1). A set of zones constitutes a subsystem of the interconnected power network. The ROCOF $d f / d t$ can be computed by subsystem $s$ and by hour $h$ :

$$
\frac{d f(h)}{d t_{s}}=\frac{f_{0} \Delta P(h)}{2 E_{k, s}},
$$

where $E_{k}$ is the kinetic energy of the subsystem, $f_{0}$ is the nominal frequency, and $\Delta P_{h}$ is an imbalance that can occur in the subsystem at hour $h$.

We focus only on ROCOF as it describes the immediate and instantaneous response of the system. What happens next depends on the load sensitivity to frequency, the generators' primary control time response, the primary reserve, the generating units protection design and settings, the defense plan design and settings.

To rank the split subsystems, the ROCOF values are normalized using the maximum absolute ROCOF value $\left|\frac{d f(h)}{d t}\right|_{\text {max }}$. The maximum absolute ROCOF is selected as the maximum obtained from the computation in one scenario or in all for comparison reasons. Therefore, the system split indicator SSI used to rank the split lines is defined as:

$$
S S I=\frac{\frac{d f(h)}{d t} s_{s}}{\left|\frac{d f(h)}{d t}\right|_{\max }} .
$$

The SSI indicator is positive in case of over-frequency and negative in the case of under-frequency phenomena.

\section{B. Methodology and process description}

The system split methodology focuses on identifying and characterizing the consequences of the theoretical system splits in large interconnected power systems, with reference to the planning phase, where all the electrical characteristics are not fully known. The starting point is 


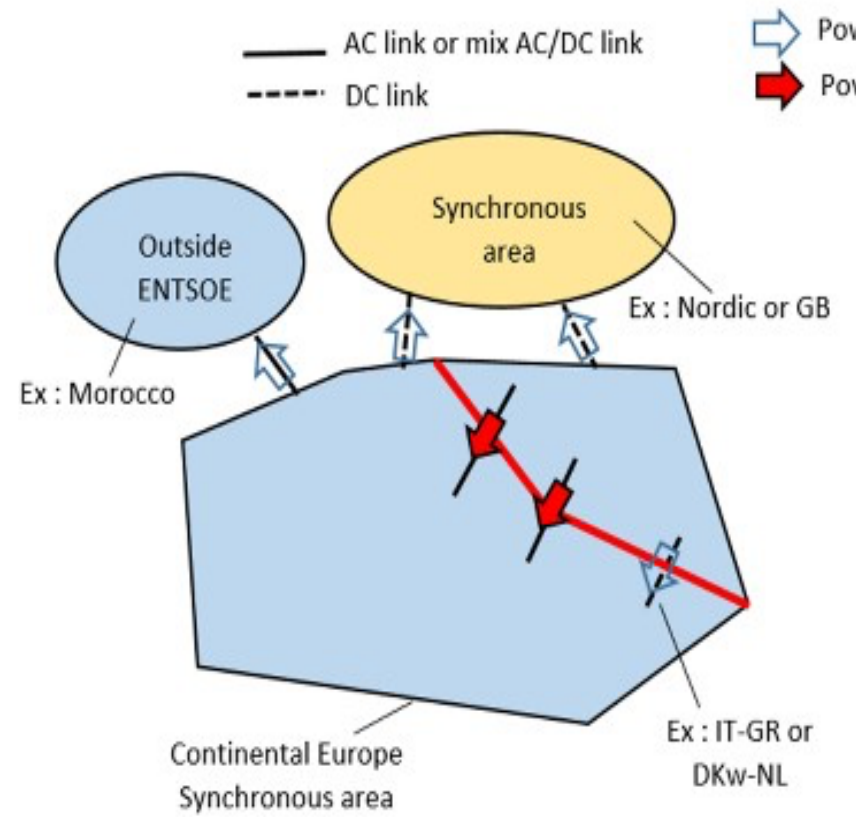

Power Flow not considered in the imbalance for the ROCOF

Power Flow considered in the imbalance for the ROCOF

Fig. 3. Details of the considered power flows for the imbalance evaluation.

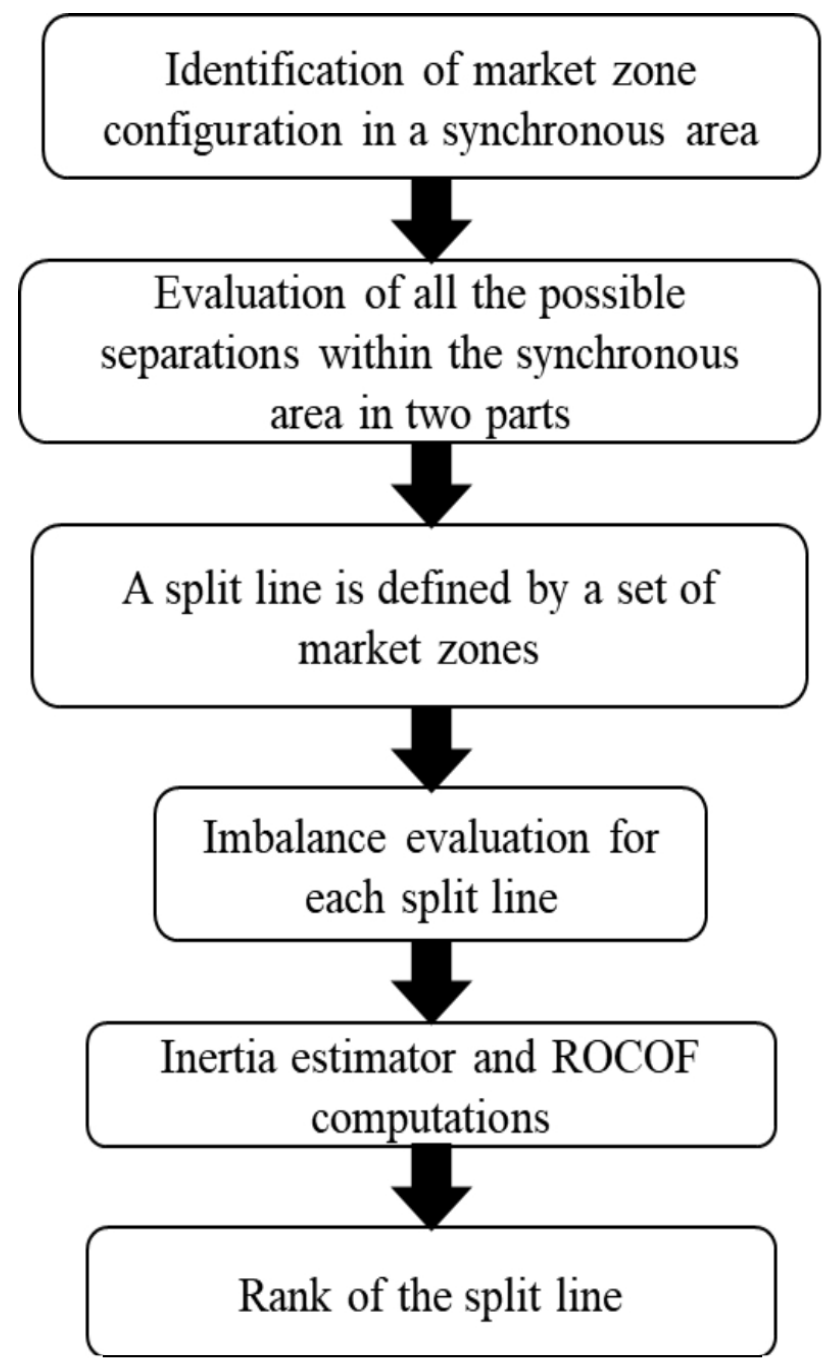

Fig. 4. Split identification methodology. the definition of the market zones, i.e., the areas within which market participants can exchange energy without capacity allocation. If the number of market zones is very large, it can be possible to wisely aggregate them for implementation reasons. Having all the market zones, it is possible to evaluate their possible separation in two or more parts. The splitting cuts are searched recursively, from each node, extending with neighboring nodes connected through AC links or AC+DC links and ensuring the cut creates only connected sets. HVDC links within a synchronous area are ignored in the computation of splitting cuts. It is assumed that the HVDC link would remain in service after the split, which is confirmed from other past major blackouts [23]. The split line is defined by a set of market zones, and the power imbalance is evaluated through each split line. Knowing the imbalance and the sets of market zones, it is possible to evaluate the inertia and running capacity in each set and calculate the frequency performance indicators.

The imbalance of an area is the sum of the AAC flows going out of the area towards the rest of the synchronous area (except if the link is purely HVDC). When two split areas within a synchronous area are connected via a purely DC link, the flows are ignored in the imbalance: it is as if the HVDC were replaced by two loads. When there is an AC link with a node outside the synchronous area, it is ignored in the imbalance: it is as if the link were replaced by two loads (Fig. 3).

The rank of the split line is made using the SSI, defined in (7). The overall process is depicted in Fig. 4.

\section{CASE STUdY}

The proposed methodology has been applied to the CE synchronous area in 2030 and 2040 horizons, coming from the TYNDP 2018, which comprises 26 countries. The 


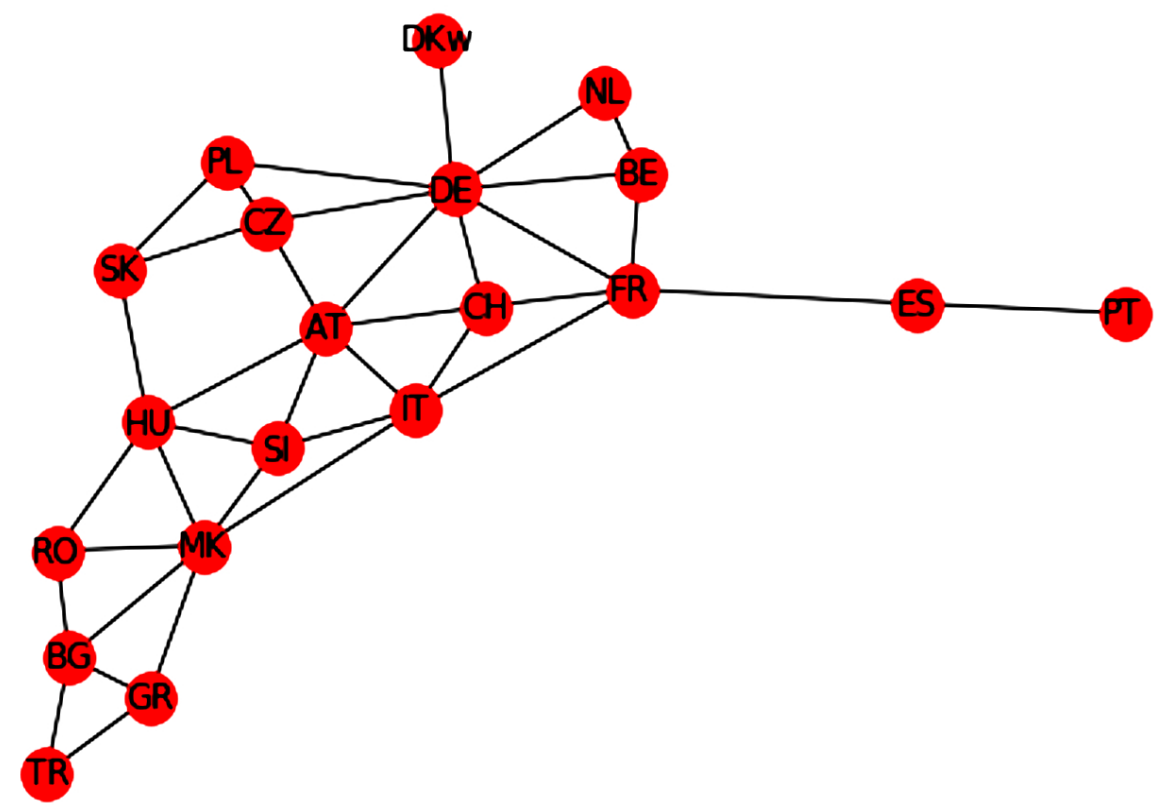

Fig. 5. Graph of the considered market zones in $C E$.

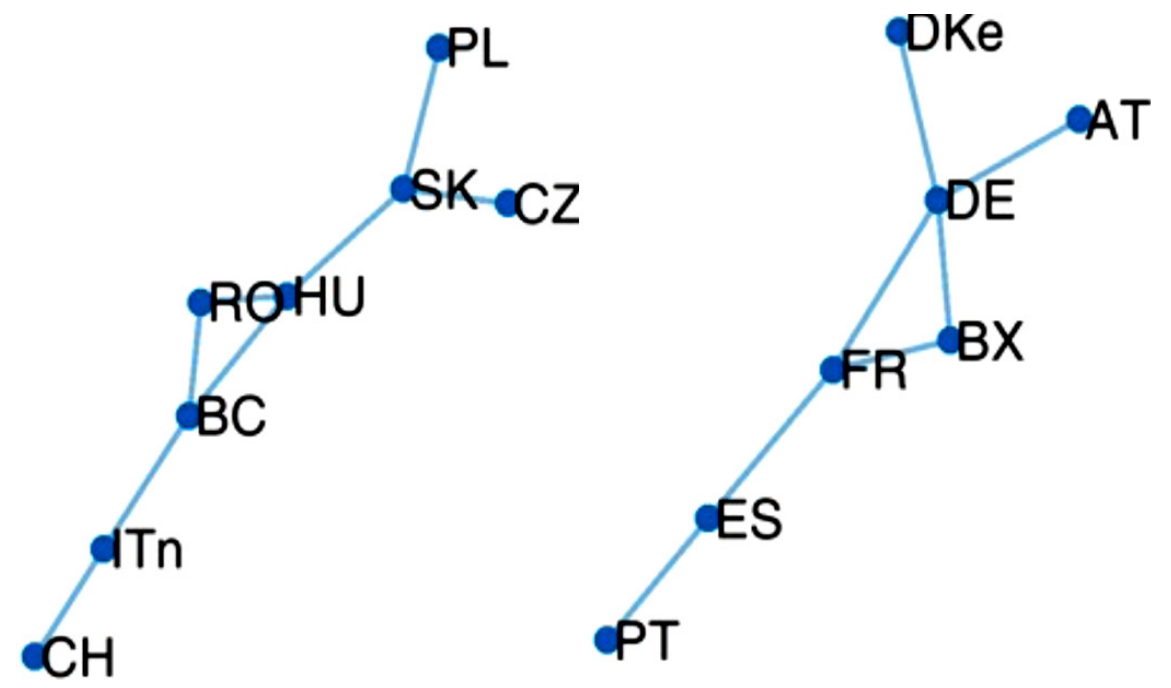

Fig. 6. Split asynchronous areas.

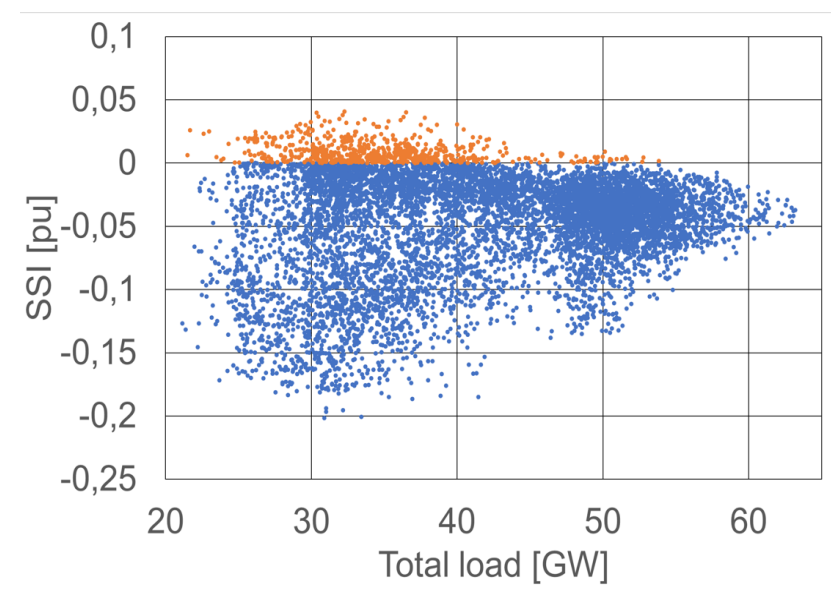

a.

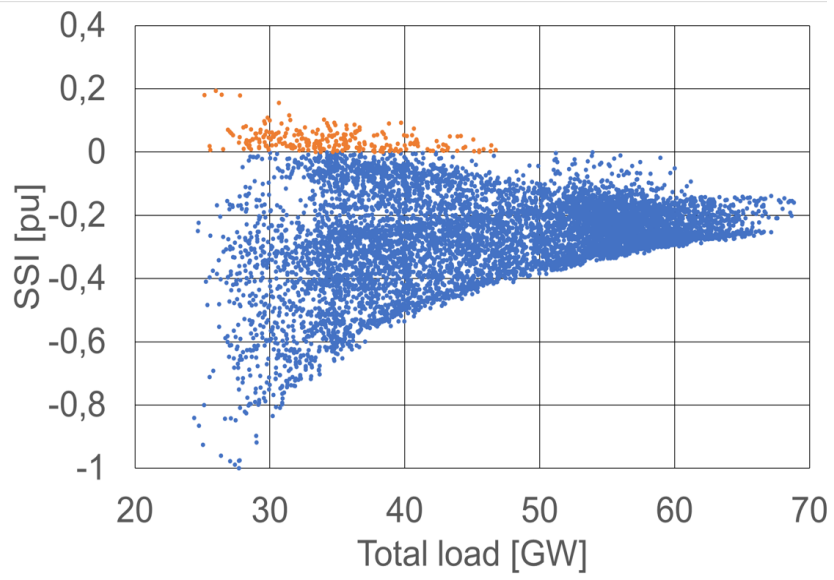

b.

Fig. 7. SSI values vs total load for all hours for the Italian subsystem separated from CE (a. ST 2030, b. GCA 2040). 
Table 1. Merged Market Zones for Computational Reasons

\begin{tabular}{ccc}
\hline \hline Node & Synchronous Area & Market zones \\
\hline $\mathrm{BE}$ & $\mathrm{CE}$ & {$[$ ['BE', 'LUb','LUg', ] } \\
\hline $\mathrm{DE}$ & $\mathrm{CE}$ & ['DE', 'LUv', 'LUg'] \\
\hline FR & $\mathrm{CE}$ & ['FR', 'LUf'] \\
\hline $\mathrm{IT}$ & $\mathrm{CE}$ & ['IT', 'ITcn', 'ITCO', 'ITcs', 'ITN', 'ITS', 'ITsar', 'ITsic', 'MT'] \\
\hline $\mathrm{MK}$ & $\mathrm{CE}$ & ['AL', 'BA', 'HR', 'ME', 'MK', 'RS'] \\
\hline \hline
\end{tabular}

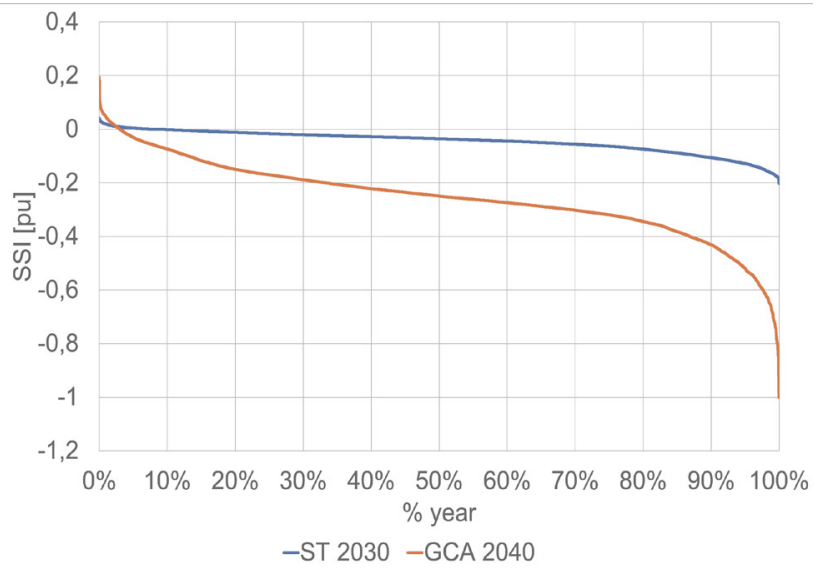

Fig. 8. SSI duration curve for all hours for the Italian subsystem separated from CE (ST 2030, GCA 2040).

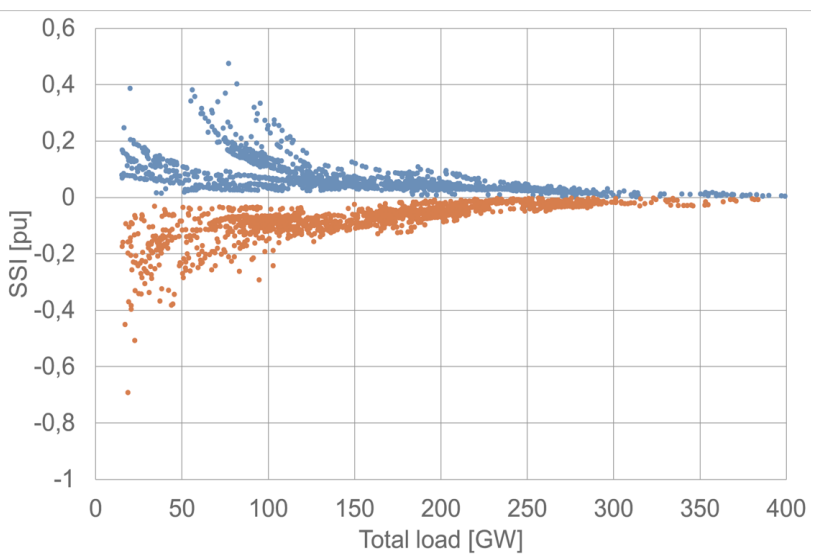

a. ST 2030

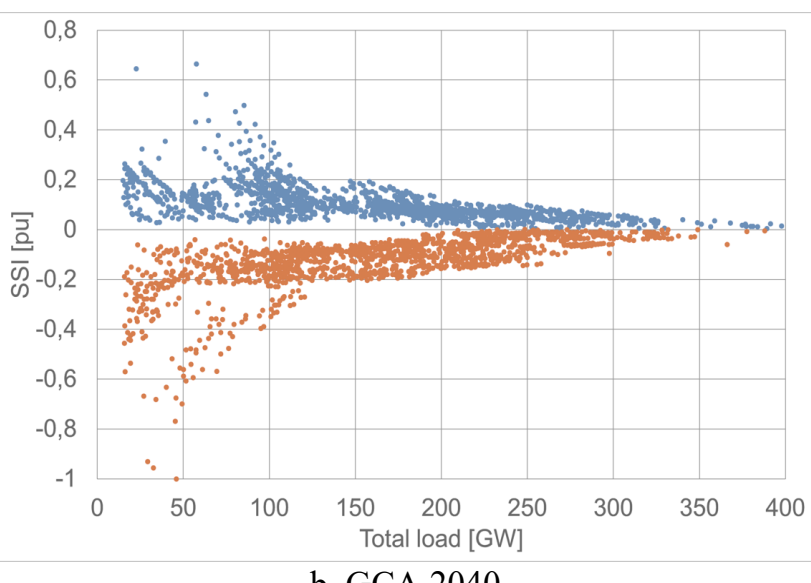

b. GCA 2040

Fig. 9. SSI values for subsystems with a total load higher than $15 \mathrm{GW}$ with respect to the total load. considered scenarios are Sustainable Transition (ST) 2030 simulated by Plexos and Global Climate Action (GCA) 2040 and simulated by PROMED, both for the climatic year 2007 [28].

The market model considers the 32 market zones reduced to 20 nodes for computational reasons. They are shown in Fig. 5 using the graph framework.

Table 1 indicates the merged market zones. Smaller market zones have been aggregated (Balkan countries), small structural antennas have been aggregated to bigger nodes (Luxembourg), and the Italian market zones are in a unique one.

All the theoretical bisections are found using the process described in Section II. Considering 20 market zones, 730 valid partitions of CE are obtained. An example of two split areas is given in Fig. 6. For each split line, the imbalance has been calculated by summing the AAC flows.

The inertia studies are performed on eleven types of units: - Nuclear • Lignite - Coal • Gas • Oil • Hydro • Wind - Solar • Other Renewable • Battery • Bio. These unit types are further divided into 44 sub-units based on technology type. Each subcategory has its parameters for inertia constant and average capacity. The inertia study is a post process of the market simulation output, which is then combined with the inertia parameters by fuel type collected and provided by the TSOs.

The values of the SSI have been calculated for all the possible subsystems and for each hour of the year. First, the case for only one subsystem is presented (the Italian area separated from $\mathrm{CE}$ ), followed by the values for all subsystems, filtered by the area size.

All the computed SSI values for Italy separated from $\mathrm{CE}$ are plotted in Fig. 7 with respect to the subsystem total load. Separated situations are identified for over-frequency and under-frequency using different colors (orange for over-frequency and blue for under-frequency). To compare the two scenarios, the SSI values for the Italian subsystem separated from the rest of Europe are referred to the maximum absolute ROCOF in both the ST 2030 and GCA 2040. In the ST 2030 scenario, the worst under-frequency situation is at hour $1680(S S I=-0.20)$ with an imbalance around $13 \mathrm{GW}$ and a total load around $31 \mathrm{GW}$. The worst over-frequency situation is at hour 8555 with $S S I=0.04$, due to an imbalance around $7 \mathrm{GW}$ and a total load around 32 GW. In the GCA 2040 scenario, the worst underfrequency situation is at hour $2670(S S I=-1)$, with an imbalance around $14 \mathrm{GW}$ and a total load around $28 \mathrm{GW}$. 
Table 2. Ranking of worst ten split lines

\begin{tabular}{|c|c|c|c|c|}
\hline & \multicolumn{2}{|c|}{ ST 2030} & \multicolumn{2}{|l|}{ GCA 2040} \\
\hline & \multicolumn{4}{|c|}{ Under-frequency } \\
\hline & Split line & $S S I[\mathrm{pu}]$ & Split line & $S S I[\mathrm{pu}]$ \\
\hline 1 & AT_CH_SI & -0.69 & AT_IT & -1 \\
\hline 2 & BE_NL & -0.51 & CH_IT & -0.96 \\
\hline 3 & AT_HU & -0.45 & IT_SI & -0.93 \\
\hline 4 & AT_CH_HU & -0.40 & AT_IT_SI & -0.77 \\
\hline 5 & AT_CZ & -0.38 & AT_CH_IT & -0.70 \\
\hline 6 & AT_CH_IT & -0.38 & CH_IT_SI & -0.68 \\
\hline 7 & AT_IT & $\begin{array}{l}-0.38 \\
\end{array}$ & GR_IT_MK & -0.68 \\
\hline 8 & AT_HU_SI & -0.37 & BE_NL & -0.67 \\
\hline 9 & $\mathrm{CH} \_\mathrm{IT}$ & -0.37 & IT_MK & -0.63 \\
\hline \multirow[t]{2}{*}{10} & AT_CH_IT_SI & -0.34 & AT_CH_IT_SI & -0.61 \\
\hline & \multicolumn{4}{|c|}{ Over-frequency } \\
\hline 1 & DE_DKw & 0.48 & DE_DKw & 0.66 \\
\hline 2 & DE_DKw_NL & 0.40 & BE_NL & 0.64 \\
\hline 3 & BE_NL & 0.39 & CH_DE_DKw & 0.54 \\
\hline 4 & AT_DE_DKw & 0.38 & DE_DKw_NL & 0.50 \\
\hline 5 & CH_DE_DKw & 0.37 & AT_DE_DKw & 0.47 \\
\hline 6 & AT_DE_DKw_SI & 0.36 & CZ_DE_DKw & 0.44 \\
\hline 7 & CZ_DE_DKw & 0.34 & BE_DE_DKw_NL & 0.43 \\
\hline 8 & BE_DE_DKw_NL & 0.34 & AT_DE_DKw_SI & 0.43 \\
\hline 9 & CH_DE_DKw_NL & 0.33 & CH_DE_DKw_NL & 0.42 \\
\hline 10 & AT_DE_DKw_NL & 0.32 & AT_CH_DE_DKw & 0.39 \\
\hline
\end{tabular}

The worst over-frequency situation is at hour 8571 with $S S I=0.19$, due to an imbalance around $8 \mathrm{GW}$ and a total load around $26 \mathrm{GW}$. As seen in Fig. 7, there is a slight trend towards higher values of ROCOF with low load situations.

The results are plotted in Fig. 8 as duration curves for all the hours of the year for the Italian subsystem separated from the rest of Europe, for two scenarios - ST 2030 and GCA 2040. As Italy imports for most hours of the year, the worst situation is for under-frequency phenomena, with negative SSI values for 8472 and 7986 hours, respectively, in the ST 2030 and GCA 2040.

The situation is increasingly worsening moving towards 2040. The maximum absolute value of ROCOF is for the scenario GCA 2040 in under-frequency. The worst under-frequency SSI for ST 2030 is -0.2 , around $20 \%$ of the worst SSI for the GCA 2040. The worst over-frequency SSI is 0.04 for ST 2030 and 0.19 for GCA 2040.

All the cases with a subsystem load higher than $15 \mathrm{GW}$ are filtered out. $15 \mathrm{GW}$ is considered here as suitable to identify large power system areas possibly affected by dangerous splits. Fig. 9 shows the values of the SSI for the two analyzed scenarios plotted with respect to the total subsystem load. The SSI is calculated using the maximum absolute ROCOF for all considered subsystems and scenarios.

In ST 2030, 2864 points are found, while in GCA 2040 - 2863 points, with 593 splits and 730 splits. The worst under-frequency case is the separation of AustriaSwitzerland-Slovenia at hour 358, with an SSI of - 0.69, due to an imbalance around $11 \mathrm{GW}$ and a total load of 18.6 GW in the scenario ST 2030, while the separation of Austria-Italy appears at hour 7, with an $S S I$ of $-1 \mathrm{~Hz} / \mathrm{s}$, due to an imbalance around $20 \mathrm{GW}$ and a total load of $46 \mathrm{GW}$ in the scenario GCA 2040. The worst over-frequency case is the separation of Germany-Denmark at hour 4258, with an $S S I$ of $0.47 \mathrm{~Hz} /(\mathrm{GWs})$, due to an imbalance around 24 GW and a total load of $77 \mathrm{GW}$ in the scenario ST 2030. In the scenario GCA 2040, the worst over-frequency case is the same separation of Germany-Denmark at hour 4530, with a system split indicator of 0.66 , due to an imbalance around $26 \mathrm{GW}$ and a total load around $58 \mathrm{GW}$. Table 2 indicates the ranking of the worst ten split lines for the cases of under- and over-frequency per scenario.

\section{CONCLUSION}

This paper has presented and assessed a methodology to identify large-scale power system splits into subsystems and evaluated their frequency stability. The system is considered to be composed of market zones, and all the possible separations in two synchronous areas are found using a graph approach. A set of parameters considering the inertia, the running capacity of the separated areas, and the imbalances among them, are used to evaluate the ROCOF and the system split indicator from the output of market simulations at different planning horizon and timeframes. The methodology has been tested on the simulations coming from the TYNDP 2018 scenarios of ENTSO-E. The results suggest interesting findings on the identification of possible dangerous split lines to monitor already in the planning phase. They also show that the ROCOF values above $2 \mathrm{~Hz} / \mathrm{s}$ could be verified across the spectrum of system split cases and in subsystem areas with load size larger than $20 \mathrm{GW}$. The trend from the analyzed scenarios ST 2030 to GCA 2040 indicates that the situation might worsen due to the increasing penetration of power electronics generation not contributing to the total system 
inertia. It is important then to further look at sustainable mid-term and long-term mitigation measures for reduced inertia and possible splits to guarantee the frequency stability of the power system in terms of grid reinforcement, regulations, or protection schemes. The identification of possible splits depends on several factors as the aftermath of cascading outages, including the failure or misbehavior of protections and depending on the power transfers. These aspects will be a focus of future developments of this work, together with further indicators to rank the split lines (for example, considering the probabilities of separation), and the analysis of the worst situations in detailed dynamic simulations.

\section{ACKNOWLEDGMENT}

The authors gratefully acknowledge the contributions and work of all the members of the Drafting Team Planning Standards at ENTSO-E.

\section{REFERENCES}

[1] European Commission. Clean Energy for all Europeans, 2019.

[2] J. Fang, H. Li, Y. Tang, F. Blaabjerg, "On the Inertia of Future More-Electronics Power Systems," IEEE Journal of Emerging and Selected Topics in Power Electronics, vol. 7, no. 4, pp. 2130-2146, Dec. 2019.

[3] F. Conte, S. Massucco, M. Paolone, G.P. Schiapparelli, F. Silvestro, Y. Zuo, "Frequency stability assessment of modern power systems: Models definition and parameters identification," Sust. Energy, Grids and Networks, vol. 23, 2020, art. 100384.

[4] European Commission. Commission Regulation (EU) 2017/1485 - Establishing a guideline on electricity transmission system operation, 2017.

[5] C. Mosca, F. Arrigo, A. Mazza, E. Bompard, E. Carpaneto, G. Chicco, P. Cuccia, "Mitigation of frequency stability issues in low inertia power systems using synchronous compensators and battery energy storage systems." IET Generation, Transmission \& Distribution, vol. 13, no. 17, pp. 3951-3959, Jul. 2019.

[6] C. Mosca, E. Bompard, G. Chicco, B. Aluisio, M. Migliori, C. Vergine, P. Cuccia, "Technical and Economic Impact of the Inertia Constraints on Power Plant Unit Commitment," IEEE Open Access Journal of Power and Energy, vol. 7, pp. 441-452, Oct. 2020.

[7] ENTSO-E. Frequency Stability Evaluation Criteria for the Synchronous Zone of Continental Europe, 2016.

[8] UCTE. Final Report System Disturbance on 4th November 2006, 2007.

[9] G. Xu, V. Vittal, "Slow Coherency Based Cutset Determination Algorithm for Large Power Systems," IEEE Trans. Power Syst., vol. 25, no. 2, pp. 877-884, May 2010.

[10] N.I. Voropai, “Generalized Technology of Hierarchical Modeling of Complex Energy Systems," Energy Systems Research, vol. 2, no. 4, pp. 22-25, 2019.

[11] G. Chicco, P. Colella, A. Griffone, A. Russo, Y. Zhang, E.M. Carlini, M, Caprabianca, F. Quaglia, L. Luzi,
G. Nuzzo, "Overview of the Clustering Algorithms for the Formation of the Bidding Zones," 2019 54th International Universities Power Engineering Conference (UPEC), Bucharest, Romania, Sep. 2019.

[12] T. Brouhard, M. Hennebel, M. Petit, C. Gisbert, "Bidding Zones of the European Power System: Benefits of a Multi-Dimensional Approach to the Evaluation of Possible Delineations," 2020 17th International Conf. on the European Energy Market (EEM), 2020.

[13] C.G. Wang, B.H. Zhang, Z.G. Hao, J. Shu, P. Li, Z.Q. Bo, "A Novel Real-Time Searching Method for Power System Splitting Boundary," IEEE Trans. Power Syst., vol. 25, no. 4, pp. 1902-1909, Nov. 2010.

[14] B. Rozel, R. Caire, N. Hadjsaid, J. Rognon, C. Tranchita, "Complex network theory and graph partitioning: Application to large interconnected networks," 2009 IEEE Bucharest PowerTech, Bucharest, 2009, pp. 1-6.

[15] C. Cieslak, J. Massmann, C.O. Their, A. Schnettler, "Identification of System Split Topologies in Transmission Grids," in 2018 53rd Intern. Univ. Power Engin. Conf. (UPEC), Sep. 2018, pp. 1-6.

[16] ENTSO-E. TYNDP 2018 Executive Summary, 2018.

[17] ENTSO-E. TYNDP 2018 Identification of the system needs methodology, 2019.

[18] ENTSO-E. Definitions of Transfer Capacities in liberalized Electricity Markets, 2001.

[19] ENTSO-E. Methodology: Frequency Stability Studies, 2019.

[20] ENTSO-E. European Power System 2040 Completing the map, 2019.

[21] ENTSO-E. TYNDP 2020 System dynamic and operational challenges, 2020.

[22] G. Andersson, P. Donalek, R. Farmer, N. Hatziargyriou, I. Kamwa, P. Kundur, N. Martins, J. Paserba, P. Pourbeik, J. Sanchez-Gasca, R. Schulz, A. Stankovic, C. Taylor, V. Vittal, "Causes of the 2003 major grid blackouts in North America and Europe, and recommended means to improve system dynamic performance," IEEE Trans. Power Syst., vol. 20, no. 4, 2005.

[23] UCTE. Final Report of the Investigation Committee on the 28 September 2003 Blackout in Italy, 2004.

[24] ENTSO-E. Report on blackout in Turkey on 31st March 2015, 2015.

[25] A. Martins et al., "Lessons learned in restoration from recent blackout incidents in Brazilian power system," in Proc. CIGRE Paris Conf., Paris, France, 2012, pp. $1-9$.

[26] V. Rampurkar P. Pentayya, H. A.Mangalvedekar, and F. Kazi, "Cascading failure analysis for Indian power grid,” IEEE Trans. Smart Grid, vol. 7, no. 4, pp. 19511960, Jul. 2016.

[27] C.D. Vournas, V.C., Nikolaidis, A.A. Tassoulis, "Postmortem analysis and data validation in the wake of the 2004 Athens blackout," IEEE Trans. Power Syst., vol. 21, no. 3, pp. 1331-1339, Aug. 2006.

[28] ENTSO-E. TYNDP 2018 Scenario Report, 2018. 


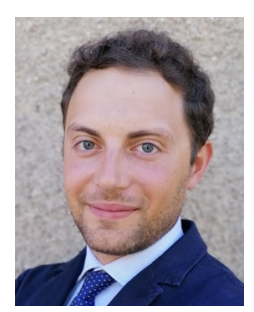

Carmelo Mosca received the M.Sc. degree in electrical engineering (with honors) from Politecnico di Torino (POLITO), Turin, Italy. He is currently pursuing the Ph.D. in Electrical, Electronics, and Communication Engineering from POLITO. His research interests include power system operation and planning, power system dynamics, data analytics, and renewable energy integration.

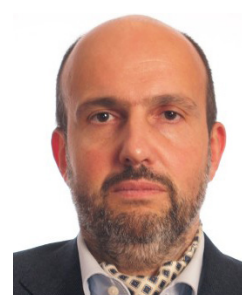

Ettore Bompard received the Ph.D. in electrical engineering from Politecnico di Torino, Turin, Italy. He is presently Professor of Power Systems at the Department of Energy of Politecnico di Torino and scientific director of the Energy Security Transition Lab at the Energy Center, Torino. His research interests include electricity markets analysis and simulation, smart grids design and modeling, power system vulnerability assessment and security management, energy security, sciencebased support to policy decision making, and data analytics applications to power systems.

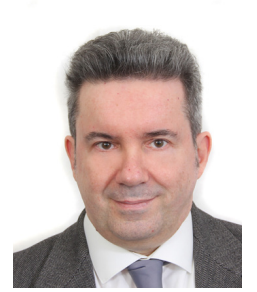

Gianfranco Chicco received the Ph.D. degree in Electrotechnics Engineering from Politecnico di Torino (POLITO), Turin, Italy. He is a Full Professor of Electrical Energy Systems at POLITO. His research interests include power system and distribution system analysis and optimization, multi-energy system optimization and planning, electrical load management, data analytics, and artificial intelligence applications to energy systems.

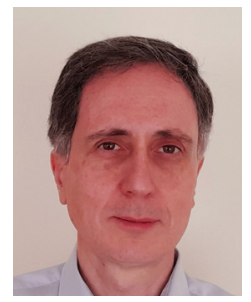

João Moreira graduated in Electrical and Computer Engineering, Energy Systems from Instituto Superior Técnico da Universidade Técnica de Lisboa, Lisbon, Portugal. He is a senior engineer in the Planning Area at REN (National Energy Networks). His background experience includes power system dynamics analysis, planning and grid development, the grid user technical requirements, and TSO cooperation work under ENTSO-E scope.

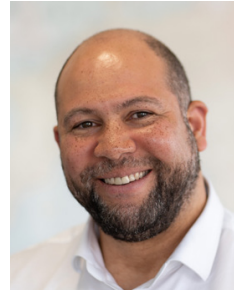

Vincent Sermanson graduated from the French Ecole Supérieure d'Electricité (Power Systems Department) in 1996. He worked for two years at the University of Liège, Belgium, and for one year at EPRI, Palo Alto, USA. After being in charge of dynamic simulation tools and studies in EDF then RTE, he is now a senior planning study engineer and system dynamics expert at RTE.

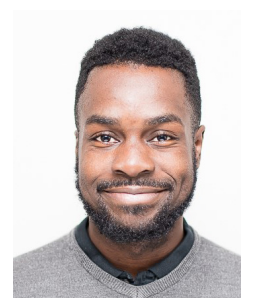

Dante Powell received the M.Sc. degree in energy systems and thermal processes from Cranfield University, Cranfield, UK. He is currently a longterm planning senior specialist at ENTSO-E and principal market modeler for the ENTSOs' scenarios. His research interests include power system planning and analysis, scenario building, energy transformation, and infrastructure development. 